\title{
Associative Strength and Activities of Motor Units in Diaphragm, Intercostal and Latissimus Dorsi during Forceful and Normal Respiration of 15-20 Years Young Male Athletes and Non- Athletes.
}

\author{
Priya Nandy ${ }^{1}$, Soumen Bhattacharyaa ${ }^{1}$, Shilpa Dutta ${ }^{1}$, Anupam Bandyopadhyay ${ }^{2 *}$ \\ ${ }^{I}$ M.Sc, Serampore College, West-Bengal, India \\ ${ }^{2}$ Associate Professor, Serampore College, West-Bengal, India
}

*Corresponding Author: Anupam Bandyopadhyay, Associate Professor, Serampore College, WestBengal, India

\begin{abstract}
An increase in respiratory activities of athletes was due to increased chest cavity by influencing the neuromuscular activities of inspiratory muscles. Physical training, whether influencing the motor units or not, was the major concern of this study. This study also focuses on the strength of association in motor units, during forceful and normal respiration of 15-20 years young male athletes and nonathletes. One way ANOVA, Scheffe's multiple comparison tests and, Strength of association were calculated. Significant differences were found between motor unit activity of three muscles in forceful and normal respiration. Scheffe's multiple comparison tests show significant differences between Intercostal and Diaphragm, Diaphragm and Latissimus dorsi muscles in both groups during both conditions. Strength of association between motor units of respiratory muscles is lower in athletes in both conditions. Athletes produce better force output with the help of small motor unit recruitment which can minimize the amount of fatigue. The duration of electromyographic bursts and strength of association of motor units are less in athletes, but motor unit activity is higher than non-athletes. This might be due to physical training because long term physical activity could improve respiratory efforts by increasing the chest cavity through the inspiratory muscles activities.
\end{abstract}

Keywords: Motor units, Respiratory muscles, Surface EMG, Normal respiration, Forceful respiration, Athletes, Nonathletes.

\section{INTRODUCTION}

In the areas of pulmonary and exercise physiology, the role of respiratory muscle is an important issue of interest. Considerable progress has been made in quantifying the capacity of the respiratory muscles in terms of strength, endurance, and fatigue [1]. Physical training enhances health in many ways. The respiratory system is greatly influenced by exercise training [2]. The respiratory muscles are specialized muscles that contract physically throughout life to maintain ventilation.

The mechanical action of the respiratory muscles is determined (like all skeletal muscles) by the bony structure(s) to which they attach, and the displacement of these structures when the muscles contract. The inspiratory pump muscles, with anatomical origins and insertions on the rib cage and spine, displace the rib cage and abdominal wall. The diaphragm is the principal muscle of inspiration $[3,4]$, but the intercostal and latissimus dorsi muscles are also active in every breath [5,6]. During quiet breathing, the timing and amount of activity in these muscles are not uniform [7,8]. If the inspiratory load or the drive to breathe increases, other skeletal muscles can be recruited as accessory muscles of inspiration [9].

Forced breathing is an active mode of breathing that utilizes additional muscles to rapidly expand and contract the thoracic cavity volume. It most commonly occurs during exercise. During exercise, the increased ventilatory demands determine an increased neural drive to the respiratory muscles. This determines an increased mechanical power developed by the muscles. Differently than rest during exercise, the diaphragm is primarily a 'flow generator'. This means that its mechanical power is 
mainly expressed as the velocity of shortening rather than pressure. During exercise, the expiratory muscles play an active role in breathing. Within every single breath, their action is highly coordinated with that of the inspiratory rib cage muscles.

Long term physical exercise leaves an effect on the diaphragm and intercostal muscles. It helps to strengthen the muscles. The respiratory volume goes up immediately and regular exercise leads to an increase in the overall efficiency of the respiratory system. It can help to increase the number of capillaries around the alveoli and helps the capillary to dilate more, which in turn facilitates the efficient exchange of gases [10].

Every day, we have taken a breath several times, typically without consciousness and with major interruptions, even while we sleep. It is very simple yet taking a breath is a complex motor function that relies on the exquisite coordinated neural activation of some skeletal muscles that generate pressure to draw air into the lungs [11]. Breathing occurs unconsciously and is controlled by a complex neural network with a final output of phrenic motor neuron activating diaphragm muscle fibre. Neuromotor control of respiratory muscles depends on the function of motor units comprising the muscle. Conditions leading to declining motor unit numbers and alteration of motor unit sizes may impact upon many aspects of neuromuscular function and physical performance. Motor unit recruitment refers to the activation of additional motor units to accomplish an increase in contractile strength in a muscle. The activation of more motor neurons will result in more muscle fibres being activated, and therefore a stronger muscle contraction.

Unloading the respiratory muscles during exercise by using low-density gas mixtures, mechanical ventilators or supplemental oxygen is neither practicable nor allowed for healthy athletes. What can be done to improve the fatigue resistance and mechanical efficiency of respiratory muscles is physical training. Although there is still no definitive evidence as to whether it is possible to improve exercise tolerance, reliable recent studies showed that respiratory muscle training has a small but probable and significant effect on endurance training performance: relief of respiratory muscle fatigue; attenuation of respiratory muscle metaboreflex; and relief of discomfort associated with high levels of respiratory muscle work [12-14].

Studies demonstrated that the use of surface electrode EMG (sEMG) is beneficial in the study of estimating the analysis of muscle activities [15]. Utilizing a non-invasive method to distinguish the type of inspiratory muscle fatigue is clinically important because it helps clinicians to design effective treatment interventions for the patients. It has long been known that externally applied electrical currents can stimulate nerves and can be used to evaluate peripheral neuromuscular function [16].

In this study surface elecrtomyographic activity of athletes and non-athletes were analyzed during forceful and normal respiration. The main purpose of this study is to focus on the motor unit activity and strength of association in motor units of inspiratory muscles during forceful and normal respiration. The hypothesis was based on the fact that exercise or physical training can increase respiratory muscle activity with the involvement of neuromuscular activities. It also helps to expand the chest cavity. Three inspiratory muscles (diaphragm, intercostal and latissimus dorsi) were the major concern of this study during both forceful and normal respiration. This research was done to conclude that the betterment of motor unit activation can be done through proper physical exercise of respiratory muscles.

\section{MATERIALS AND METHODS}

Participants: Fifty-nine (59) young male athletes and Forty-nine (49) non-athletes without a physical disability with age ranges of 15-20 years have participated in this study. All the subjects were informed in detail about the purposes of the study and the methods used in this investigation. Consent letter before enrolment of each subject was taken. Prior Ethical clearance was taken from the Institutional Human Ethics Committee according to the guidelines of the Indian Council of Medical Research.

Materials: The materials used in the current study are Surface Electromyogram (sEMG), Silver silver chloride electrode, and Spirometer.

Procedure: Surface EMG recordings of the diaphragm, external intercostal, and latissimus dorsi were obtained by using pairs of skin-taped silver/silver chloride electrodes ( $8 \mathrm{~mm}$ in diameter) filled with 
Associative Strength and Activities of Motor Units in Diaphragm, Intercostal and Latissimus Dorsi during Forceful and Normal Respiration of 15-20 Years Young Male Athletes and Non-Athletes.

conductive paste placed on the cleaned, abraded skin. After placement of sEMG leads Forceful and normal respiration was performed with the help of a Spirometer (Schiller SP1).

Statistical analysis: EMG data were analyzed off-line using the software of the machine (iworx) installed on the computer. Statistical calculations were done with the help of IBM SPSS v.24. One way ANOVA was performed among the three respiratory muscle groups $(p<0.05)$. Omega square $\left(\omega^{2}\right)$ is computed to estimate the strength of association between the motor unit activity of respiratory muscles and forceful and normal respiration.

\section{RESUltS}

Mean and Standard deviation (SD) of physical parameters (age, height, weight) of Athletes and Nonathletes were represented in Table $\mathbf{1}$.

Table1. Mean $\pm S D$ value of physical parameters of Athletes and Non-athletes group.

\begin{tabular}{|l|l|l|}
\hline Parameters & Athletes $(\mathbf{n}=\mathbf{5 9})$ & Non-athletes $(\mathbf{n}=\mathbf{4 9})$ \\
\hline Age (years) & $15.96 \pm 2.7$ & $18.53 \pm 1.06$ \\
\hline Height $(\mathbf{c m})$ & $166.7 \pm 7.05$ & $169.42 \pm 5.94$ \\
\hline Weight $(\mathbf{k g})$ & $55.52 \pm 8.61$ & $60.24 \pm 9.29$ \\
\hline
\end{tabular}

Table 2 shows the mean and SD of surface electromyographic activity of three different respiratory muscles (Intercostal, Diaphragm, Latissimus dorsi) of athletes and non-athletes during forceful respiration. One way anova was calculated to understand the significant difference between the three groups of muscles (Table 2). Similar calculations during normal respiration were represented in Table 3. Significant differences $(p<0.05)$ were found between motor unit activity in case of both forceful and normal respiration.

Table2. Mean \pm SD value of surface electromyographic activity of respiratory muscles of Athletes and Nonathletes groups during Forceful respiration [ $n=$ Sample size, $F$ value=Analysis of variance (ANOVA) and $p=$ Level of significance $]$.

\begin{tabular}{|l|l|l|l|l|}
\hline \multicolumn{5}{|c|}{ ATHLETES (n=59) during FVC } \\
\hline Parameters & Intercostal & Diaphragm & Latissimus dorsi & F value \\
\hline Integral of EMG signal & $1.06 \pm 0.99$ & $\mathbf{1 . 2 3} \pm \mathbf{0 . 7 5}$ & $0.98 \pm 0.59$ & NS \\
\hline The duration of EMG bursts & $\mathbf{5 . 8 7} \pm \mathbf{2 . 0 2}$ & $5.67 \pm 1.64$ & $5.50 \pm 1.78$ & NS \\
\hline Motor unit activity & $0.24 \pm 0.13$ & $\mathbf{0 . 3 2} \pm \mathbf{0 . 1 5}$ & $0.26 \pm 0.14$ & $5.95, \mathrm{p}<0.05$ \\
\hline Peak Value & $0.33 \pm 0.18$ & $\mathbf{0 . 4 5} \pm \mathbf{0 . 2 1}$ & $0.36 \pm 0.20$ & $5.96, \mathrm{p}<0.05$ \\
\hline Peak to Peak & $0.67 \pm 0.37$ & $\mathbf{0 . 9 1} \pm \mathbf{0 . 4 2}$ & $0.73 \pm 0.39$ & $5.95, \mathrm{p}<0.05$ \\
\hline \multicolumn{7}{|l}{ NON-ATHLETES (n=49) } & during FVC \\
\hline Integral of EMG signal & $0.70 \pm 0.38$ & $0.86 \pm 0.40$ & $\mathbf{0 . 9 2} \pm \mathbf{0 . 7 3}$ & NS \\
\hline The duration of EMG bursts & $5.63 \pm 1.67$ & $5.62 \pm 2.03$ & $\mathbf{6 . 2 6} \pm \mathbf{2 . 8 8}$ & NS \\
\hline Motor unit activity & $0.17 \pm 0.06$ & $\mathbf{0 . 2 4} \pm \mathbf{0 . 0 9}$ & $0.23 \pm 0.12$ & $8.29, \mathrm{p}<0.05$ \\
\hline Peak Value & $0.24 \pm 0.09$ & $\mathbf{0 . 3 5} \pm \mathbf{0 . 1 2}$ & $0.32 \pm 0.17$ & $8.29, \mathrm{p}<0.05$ \\
\hline Peak to Peak & $0.48 \pm 0.18$ & $\mathbf{0 . 6 9} \pm \mathbf{0 . 2 5}$ & $0.65 \pm 0.35$ & $8.28, \mathrm{p}<0.05$ \\
\hline
\end{tabular}

Table3. Mean $\pm S D$ value of surface electromyographic activity of respiratory muscles of Athletes and Nonathletes groups during Normal respiration [ $n=$ Sample size, $F$ value=Analysis of variance (ANOVA) and $p=$ Level of significance].

\begin{tabular}{|c|c|c|c|c|}
\hline \multicolumn{5}{|c|}{ ATHLETES (n=59) during MV } \\
\hline Parameters & Intercostal & Diaphragm & Latissimus dorsi & F value \\
\hline Integral of EMG signal & $1.16 \pm .57$ & $1.75 \pm 0.79$ & $0.89 \pm 0.36$ & $32.22, \mathrm{p}<0.05$ \\
\hline The duration of EMG bursts & $12.52 \pm 3.15$ & $12.43 \pm 1.85$ & $12.75 \pm 1.82$ & $0.29, \mathrm{p}<0.05$ \\
\hline Motor unit activity & $0.13 \pm 0.07$ & $0.25 \pm 0.11$ & $0.11 \pm 0.05$ & $57.22, \mathrm{p}<0.05$ \\
\hline Peak Value & $0.18 \pm 0.10$ & $0.36 \pm 0.15$ & $0.16 \pm 0.07$ & $57.22, \mathrm{p}<0.05$ \\
\hline Peak to Peak & $0.36 \pm 0.20$ & $0.72 \pm 0.30$ & $0.32 \pm 0.14$ & $57.23, \mathrm{p}<0.05$ \\
\hline \multicolumn{5}{|c|}{ NON-ATHLETES $(n=49)$ during MV } \\
\hline Integral of EMG signal & $1.60 \pm 0.97$ & $2.19 \pm 1.27$ & $1.57 \pm 1.88$ & $2.98, \mathrm{p}<0.05$ \\
\hline The duration of EMG bursts & $23.29 \pm 10.99$ & $23.58 \pm 11.09$ & $23.86 \pm 11.35$ & NS \\
\hline Motor unit activity & $0.09 \pm 0.03$ & $0.18 \pm 0.05$ & $0.09 \pm 0.03$ & $94.20, \mathrm{p}<0.05$ \\
\hline Peak Value & $0.13 \pm 0.04$ & $0.25 \pm 0.07$ & $0.13 \pm 0.04$ & $94.33, \mathrm{p}<0.05$ \\
\hline Peak to Peak & $0.26 \pm 0.08$ & $0.50 \pm 0.13$ & $0.26 \pm 0.08$ & $94.19, \mathrm{p}<0.05$ \\
\hline
\end{tabular}

International Journal of Sports and Physical Education (IJSPE)

Page | 3 
Scheffe's multiple comparison tests were performed to understand which group means differ significantly from each other (Table 4). Here we have found that group means of Intercostal and Diaphragm, Diaphragm, and Latissimus dorsi muscles show a significant difference in both groups during both conditions. Only Intercostal and Latissimus muscles show a significant difference during forceful respiration of non-athletes.

Table4. Scheffe's Multiple Comparison Test

\begin{tabular}{|c|c|c|c|c|c|}
\hline \multirow{2}{*}{\multicolumn{2}{|c|}{ Parameters }} & \multicolumn{2}{|c|}{ During FVC } & \multicolumn{2}{|c|}{ During MV } \\
\hline & & \multirow{2}{*}{$\begin{array}{l}\text { Athletes } \\
p<0.05\end{array}$} & \multirow{2}{*}{$\begin{array}{l}\text { Non-athletes } \\
\mathrm{p}<0.05\end{array}$} & \multirow{2}{*}{$\begin{array}{l}\text { Athletes } \\
\mathrm{p}<0.05\end{array}$} & \multirow{2}{*}{$\begin{array}{l}\text { Non-athletes } \\
\mathrm{p}<0.05\end{array}$} \\
\hline Motor & Intercostal and Diaphragm & & & & \\
\hline $\begin{array}{l}\text { unit } \\
\text { activity }\end{array}$ & $\begin{array}{l}\text { Intercostal and Latissimus } \\
\text { dorsi }\end{array}$ & NS & $\mathrm{p}<0.05$ & NS & NS \\
\hline between & $\begin{array}{l}\text { Diaphragm and Latissimus } \\
\text { dorsi }\end{array}$ & $\mathrm{p}<0.05$ & NS & $\mathrm{p}<0.05$ & $\mathrm{p}<0.05$ \\
\hline
\end{tabular}

In this study, another statistical calculation was performed which is "Strength of association". It is represented by Omega square $\left(\omega^{2}\right)$. This Omega square was computed to estimate the strength of association between motor units of respiratory muscles and forceful and normal respiration. It has been found that athletes have less strength of association than non-athletes in both conditions.

Strength of association: Omega square was computed to estimate the strength of association between the motor unit of respiratory muscles and forceful and normal respiration.

Strength of association during forceful respiration of athletes: $\omega 2=\frac{(3-1)(5.95-1)}{(3-1)(5.95-1)+177}=\mathbf{0 . 0 5}$

Strength of association during forceful respiration of Non-athletes: $\omega 2=\frac{(3-1)(8.29-1)}{(3-1)(8.29-1)+147}=\mathbf{0 . 0 9}$

Strength of association during normal respiration of athletes: $\omega 2=\frac{(3-1)(57.22-1)}{(3-1)(57.22-1)+177}=\mathbf{0 . 3 9}$

Strength of association during normal respiration of Non-athletes: $\omega 2=\frac{(3-1)(94.20-1)}{(3-1)(94.20-1)+147}=\mathbf{0 . 5 6}$

\section{DISCUSSION}

Surface electromyography (sEMG) is a technique for recording and monitoring the electrical signals from muscle contractions. It is a generic term fora method of recording electrical muscle activity. Numerous applications for this method have been developed in clinical practice, such as diagnosing neuromuscular diseases, analyzing and determining abnormalities or disorders, and muscular rehabilitation [15].

The duration of sEMG activity corresponds to the duration of muscle activation. The amplitude is the level of signal activity and varies with the amount of electrical activity detected in the muscle; it provides information about the intensity of muscle activation [15].

Tables 2 and 3 show, integral of EMG activity is higher in athletes during forceful respiration. Opposite results have been found during normal respiration which shows that the integral of EMG activity of Non-athletes is greater than athletes. The duration of EMG bursts is higher in Non-athletes during both forceful and normal respiration. This means the respiratory muscles of Non-athletes take much more time to complete the contraction than athletes. The motor unit activity, Peak value, and Peak to Peak amplitude are higher in case of athletes during both forceful and normal respiration. That signifies that motor unit involvement and its function (contraction and relaxation) is very much better or improved in case of athletes. Athletes take less time than Non-athletes to complete a forceful respiration cycle and a normal respiration cycle but their muscle activity is much better than Nonathletes. So, it can be said that training can improve respiratory muscles to function properly and stay very active to respond quickly in different conditions.

The integral of EMG activity shows a higher response in Diaphragm muscle in athletes during both forceful and normal respiration. This indicates that electromyographic activity is high in the diaphragm than in other respiratory muscles. The motor unit activity, Peak value, and Peak to Peak amplitude are also higher in the Diaphragm muscle than the other two muscles in athletes as well as Non-athletes. Though the value of activation is lower in the Non-athletes group, Diaphragm is the 
highly active muscle for both groups. Here this study also proves that Diaphragm is the primary muscle of breathing in terms of motor unit activity.

The duration of EMG bursts is higher in Latissimus dorsi muscle in Non-athletes for both conditions. A similar observation was found in athletes during normal respiration. But during forceful respiration intercostal muscle of athletes contracts for a longer time than the other two. The motor unit activation is less in the Latissimus dorsi muscle than other muscles but the duration of activity is longer in this muscle.

Henneman's size principle [16] describes relationships between properties of motor neurons and the muscle fibres they innervate and thus control, which together are called motor units. Motor neurons with large cell bodies tend to innervate fast-twitch, high force, less fatigue resistance muscle fibres, whereas motor neurons with small cell bodies tend to innervate slow-twitch, low force, fatigue resistance muscle fibres. To contract a particular muscle, motor neurons with small cell bodies are recruited (i.e. begin to fire action potentials) before motor neurons with large cell bodies.

This study shows an association of motor unit activity which is lower in athletes during both forceful and normal respiration. This means that the activation of the motor units is higher for Non-athletes groups. When a motor unit is activated, all fibres present in that have started to contract. The force of a muscle contraction is controlled by the number of activated motor units. So it can be considered that to perform the forceful as well as normal respiration Non-athletes group produces much more muscle force than athletes.

As more force is needed to perform forceful respiration, motor units are recruited in a precise order according to the magnitude of their force output, with small units being recruited first, thus exhibiting task appropriate recruitment. Firstly it minimizes the amount of fatigue and secondly, the relative change in force produced by additional recruitment remains relatively constant [16]. For this reason, the motor unit association is low in athletes but the activity is much high and better (better responses in less time) than non-athletes. This may be due to long-term physical activity because physical training can improve the strength and endurance of respiratory muscles and thereby improve the respiratory system.

\section{CONCLUSION}

In this study, we have observed that strength association of motor unit is lower in athletes during both forceful and normal respiration but motor unit activity is higher in athletes for three muscles (intercostal, diaphragm, and latissimus dorsi). Athletes produce better force output with the help of small motor unit recruitment. This can minimize the amount of fatigue and the relative change in force produced by different types of respiration. With the motor unit activity, the duration of electromyographic bursts is much less in athletes than non-athletes. From this study, we can conclude that the force of a muscle contraction is controlled by the number of activated motor units. In athletes, the strength of association of the motor unit is less but motor unit activity is higher. So, with less time and with less strength of association of motor unit athletes produce better motor unit activity whereas non-athletes take more time, more strength of association of motor unit but less motor unit activity. This might be due to physical training because long term physical activity can improve respiration by increasing the flexibility of respiratory muscles.

\section{ACKNOWLEDGMENTS}

We acknowledge our sincere thanks to the Institutional Human Ethics Committee to permit us to perform this research works. We are also thankful for club authorities, coaches, and each participant.

\section{CONFLICTS OF INTEREST}

The authors claim no conflicts of interest for this research work.

\section{REFERENCES}

[1] Chien M. Y., Wu Y. T. and Ya-Ju Chang, Assessment of Diaphragm and External Intercostals Fatigue from Surface EMG using Cervical Magnetic Stimulation, Sens. J. 8(4), Pp. 2174-2187 (2008). 
[2] Bougault V., Turmel J., St-Laurent J., Bertrand M. and Boulet L. P., Asthma, airway inflammation and epithelial damage in swimmers and cold-air athletes, Eur. Respir. J. 33(4), Pp. 740-6 (2009).

[3] Agostoni E. and Sant'Ambrogio G., The diaphragm $2^{\text {nd }}$ ed. Lloyd-Luke Ltd, London, 1970, ch. The Respiratory Muscles: Mechanics and Neural Control, pp. 145-160.

[4] Aliverti A., Cala S. J., Duranti R., Ferrigno G., Kenyon C. M., Pedotti A., Scano G., Sliwinski P., Macklem P. T. and Yan S., Human respiratory muscle actions and control during exercise, J. Appl. Physiol. 83, Pp. 1256-1269 (1997).

[5] De Troyer A. and Sampson M. G., Activation of the parasternal intercostals during breathing efforts in human subjects, J. Appl. Physiol. 52, Pp. 524-529 (1982).

[6] De Troyer A. and Estenne M., Coordination between rib cage muscles and diaphragm during quiet breathing in humans, J. Appl. Physiol. 57, Pp. 899-906 (1984).

[7] Saboisky J. P., Gorman R. B., De Troyer A., Gandevia S. C. and Butler J. E., Differentialactivation among five human inspiratory motoneuron pools during tidal breathing, J. Appl. Physiol. 102, Pp. 772-780 (2007).

[8] Butler J. E. and Gandevia S. C., The output from human inspiratory motoneurone pools, J. Physiol.. 586, Pp. 1257-1264 (2008).

[9] Campbell E. J., Accessory muscles, $2^{\text {nd }}$ ed. Lloyd-Luke Ltd, London, 1970, ch. The Respiratory Muscles: Mechanics and Neural Control, pp. 181-193.

[10] https://expand-a-lung.com/effects-of-exercise-on-the-respiratory-system/

[11] Tokizane T., Kawamata K. and Tokizane H., Electromyographic studies on the human respiratory muscles; studies on the activity pattern of neuromuscular units, Jpn. J. Physiol. 2, Pp. 232-247 (1952).

[12] Larsson L., Grimby G. and Karlsson J., Muscle strength and speed of movement in relation to age and muscle morphology, J. Appl. Physiol. Respir. Environ. Exerc. Physiol. 3, Pp. 451-6. (1979).

[13] https://www.intechopen.com/books/computational-intelligence-in-electromyography-analysis-aperspective-on-current-applications-and-future-challenges/application-of-surface-electromyography-inthe-dynamics-of-human-movement.

[14] Hanneman E., Relation between size of neurons and their susceptibility to discharge, Science, 126 (3287), Pp. 1345-1347 (1957).

[15] Decramer M., Lacquet L. M., Fagard R. and Rogiers P., Corticosteroids contribute to muscle weakness in chronic airflow obstruction, Am. J. Respir. Crit. Care. Med. 150, Pp. 11-16 (1994).

[16] Lotters F., Van Tol B., Kwakkel G. and Gosselink R., Effects of controlled inspiratory muscle training in patients with COPD: a meta-analysis, Eur. Respir. J. 20, Pp. 570-576 (2002).

Citation: Anupam Bandyopadhyay, et.al. "Associative Strength and Activities of Motor Units in Diaphragm, Intercostal and Latissimus Dorsi during Forceful and Normal Respiration of 15-20 Years Young Male Athletes and Non-Athletes." International Journal of Sports and Physical Education (IJSPE), vol 7, no. 1, 2020, pp. 0106. doi: https://doi.org/10.20431/2454-6380.0701001.

Copyright: (C) 2021 Authors. This is an open-access article distributed under the terms of the Creative Commons Attribution License, which permits unrestricted use, distribution, and reproduction in any medium, provided the original author and source are credited. 